\title{
Changes in Macrophage Phenotype and Induction of Epithelial- to-Mesenchymal Transition Genes Following Acute Achilles Tenotomy and Repair
}

\author{
Kristoffer B Sugg ${ }^{1,2,3}$, Jovan Lubardic ${ }^{1,2}$, Jonathan P Gumucio ${ }^{1,2}$, and Christopher L \\ Mendias ${ }^{1,2, *}$ \\ 1Department of Orthopaedic Surgery, University of Michigan Medical School, Ann Arbor, MI \\ ${ }^{2}$ Department of Molecular and Integrative Physiology, University of Michigan Medical School, Ann \\ Arbor, MI \\ ${ }^{3}$ Department of Surgery, Section of Plastic Surgery, University of Michigan Medical School, Ann \\ Arbor, MI
}

\begin{abstract}
Tendon injuries occur frequently in physically active individuals, but the clinical outcomes for these injuries can be poor. In many injured tissues the repair process is orchestrated by two types of cells, macrophages and fibroblasts. Macrophages, which have both proinflammatory (M1) and antiinflammatory (M2) phenotypes, can directly participate in tissue remodeling and direct the response of other cells through the secretion of cytokines and growth factors. In many organ systems, epithelial cells can transdifferentiate into fibroblasts, which can then regenerate damaged ECM. This process is triggered via activation of epithelial-to-mesenchymal transition (EMT) signaling programs. Most tendons are surrounded by sheets of epithelial cells, and these tissue layers could provide a source of fibroblasts to repair injured tendons. To gain greater insight into the biology of tendon repair, we performed a tenotomy and repair in Achilles tendons of adult rats and determined changes in macrophage phenotype, and ECM- and EMT-related genes over a four week time course. The results from this study suggest that changes in macrophage phenotype and activation of EMT-related programs likely contribute to the degradation and subsequent repair of injured tendon tissue.
\end{abstract}

\section{Keywords}

tendon; macrophage; epithelial-to-mesenchymal transition; repair; fibrosis

\section{Introduction}

Tendon is a dynamic tissue that promotes efficient force transmission and drives locomotion by connecting muscle to bone. Injuries and diseases of tendons produce significant

\footnotetext{
${ }^{*}$ To whom correspondence should be addressed: Christopher L Mendias, PhD, Department of Orthopaedic Surgery, University of Michigan Medical School, 109 Zina Pitcher Place, Biomedical Science Research Building, Room 2017, Ann Arbor, MI 48109-2200, Telephone: (734) 764-3250, Fax: (734) 647-0003, cmendias@umich.edu.
} 
morbidity in patients, including debilitating pain and a decrease in functional capacity ${ }^{1}$. While the etiology of tendon injury remains unclear, the accumulation of degenerative changes in the tendon extracellular matrix (ECM) over time predisposes the patient to spontaneous tendon rupture ${ }^{1 ; 2}$. Repetitive microtrauma is thought to overwhelm the ability of tendon fibroblasts to repair the injured ECM network, and this likely leads to variegated cellularity, increased vascular elements and collagen fibril disorganization ${ }^{1 ; 3}$. Indeed, degenerative tendinopathy is the most common histological finding at the site of tendon rupture, and reversal of this process can be challenging because tendons are particularly slow to heal ${ }^{4}$. Accordingly, the management of tendon injuries is an exigent task for clinicians, and in many cases, patients remain symptomatic despite optimal therapy ${ }^{2}$. In order to improve the treatment of tendon injuries, a greater understanding of the cellular and molecular factors that drive tendon healing is required.

In many injured tissues, the repair process proceeds through three overlapping stages described as the inflammatory, proliferative and remodeling phases ${ }^{2 ;}$. Although numerous cell types play important roles in tendon healing, the repair of injured tendon tissue is thought to be orchestrated by two types of cells, macrophages and fibroblasts ${ }^{1}$. Following the initial recruitment of neutrophils to the site of injury, an accumulation of macrophages is observed in the tendon ECM within the first 24 hours ${ }^{6}$. Macrophages participate in the phagocytic removal of necrotic debris, but eventually shift their function to promote fibroblast proliferation and guide ECM remodeling through the release of chemotactic and growth factors $2 ; 5 ; 7 ; 8$. Macrophages have two general phenotypes - classically activated proinflammatory macrophages (M1) that promote ECM breakdown, inflammation and apoptosis; and alternatively activated antiinflammatory macrophages (M2) that coordinate ECM deposition and tissue repair ${ }^{9 ; 10}$. Previous work in damaged skeletal muscle has demonstrated that suppression of either M1 or M2 macrophage function severely impairs tissue regeneration, indicating the important balance between inflammation and regeneration for proper tissue repair ${ }^{11}$.

Fibroblasts are the predominant cell type in tendons and are responsible for the development, maintenance, repair and modification of the tendon ECM ${ }^{12}$. In response to tissue injury, fibroblasts synthesize ECM proteins, including collagens, proteoglycans and glycoproteins ${ }^{12}$. Fibroblasts also release matrix metalloproteinases (MMPs), a family of zinc-dependent enzymes that selectively degrade various components of the ECM network, and their endogenous inhibitors, tissue inhibitors of metalloproteinases (TIMPs) ${ }^{3}$. The levels of MMPs and TIMPs fluctuate throughout the repair process, which culminates in the eventual replacement of the damaged tendon ECM with newly synthesized collagen and noncollagenous proteins $1 ; 2 ; 12$. Previous work has demonstrated that the epitenon, a loose epithelial-like tissue layer that surrounds the tendon, may serve as a source of fibroblasts contributing to tendon growth and repair ${ }^{13 ; 14}$. In other organ systems, reactivation of one or more members of the Snail family of transcription factors promotes the transdifferentiation of epithelial cells to a mesenchymal cell lineage through a process known as epithelial-tomesenchymal transition (EMT) ${ }^{15-17}$. Once in the mesenchymal lineage, the cells adopt a fibroblast phenotype and begin remodeling and synthesizing new ECM ${ }^{15-17}$. While EMT plays an important part in the regeneration of other tissues, the role of the EMT process in tendon repair has not been previously described. 
To gain greater understanding of the fundamental cellular and molecular biology of tendon repair, we performed a tenotomy and repair in Achilles tendons of adult rats and analyzed changes in macrophage phenotype, and ECM- and EMT-related genes. Tissue was harvested at 3, 7, 14 and 28 days after injury and compared to uninjured controls. We hypothesized that in response to a full-thickness tear of the Achilles tendon there would be an early accumulation of M1 macrophages within the first week followed by gradual transition to the M2 phenotype, and that tendon repair would correlate with increased expression of EMTrelated genes.

\section{Methods}

\section{Animals and Surgical Procedure}

The University of Michigan IACUC approved this study. Six-month-old male SpragueDawley rats were randomized to control, 3-, 7-, 14- or 28-day groups ( $\mathrm{N}=6$ rats per group). Tendon surgery was performed on both Achilles tendons. Rats were anesthetized with $2 \%$ isoflurane, and the skin overlying the surgical site was shaved and scrubbed with $4 \%$ chlorhexidine. A midline incision was created in the skin and the paratenon was split to achieve visualization of the Achilles tendon. A full-thickness tenotomy was performed in the mid-substance of the tendon followed by immediate two-strand repair using the Bunnell technique with 5-0 Ethibond (Ethicon, Somerville, NJ) (Figure 1). The plantaris tendon was left intact. The paratenon was loosely reapproximated and the skin closed using 4-0 Vicryl (Ethicon) and GLUture (Abbott, Abbott Park, IL). A splash block of $0.5 \mathrm{ml}$ of $0.5 \%$ bupivacaine, buprenorphine $(0.05 \mathrm{mg} / \mathrm{kg})$ and carprofen $(5 \mathrm{mg} / \mathrm{kg})$ were administered for analgesia during postoperative recovery. Ad libitum weightbearing and cage activity were allowed. At harvest, rats were anesthetized with pentobarbital $(50 \mathrm{mg} / \mathrm{kg})$ and Achilles tendons were harvested for either gene expression analysis or immunohistochemistry (IHC). Achilles tendons were also collected from rats that did not undergo tenotomy and served as controls. After the removal of tendons, animals were euthanized by anesthetic overdose and induction of bilateral pneumothorax.

\section{Gene Expression}

The right Achilles tendon was homogenized in QIAzol (Qiagen, Valencia, CA). RNA was isolated using a miRNeasy Kit (Qiagen), treated with DNase I (Qiagen) and reverse transcribed into cDNA using oligo- $\mathrm{dT}_{15}$ and random hexamer primers with the Omniscript RT Kit (Qiagen). cDNA was amplified in a CFX96 real-time thermal cycler (Bio-Rad, Hercules, CA) using a QuantiTect SYBR Green (Qiagen). The methods of Livak and Schmittgen ${ }^{18}$ were used to normalize target gene expression to the stable housekeeping gene $\beta 2$ - microglobulin (B2M). Transcript information and the specific function of each gene are provided in Supplementary Table 1.

\section{Immunohistochemistry}

Left Achilles tendons were isolated, snap frozen in TFM (Triangle Biosciences, Durham, NC) and stored at $-80^{\circ} \mathrm{C}$. Tendons were sectioned through the callus at a thickness of $10 \mu \mathrm{m}$ in a cryostat. Slides were fixed in $4 \%$ paraformaldehyde, permeabilized in $0.2 \%$ Triton X-100 and blocked with 5\% goat serum. Slides were incubated with primary antibodies 
against rabbit anti-CCR7 (NB110-55680, Novus Biologicals, Littleton, CO) and mouse antiCD163 (MCA342B, AbD Serotec, Raleigh, NC) to label M1 and M2 macrophages, respectively ${ }^{19 ; 20}$. AlexaFluor 555 (AF555, Life Technologies, Eugene, OR) secondary antibodies or streptavidin-AF647 were used to detect primary antibodies. DAPI was used to identify nuclei and ECM was labeled with WGA-lectin conjugated to AF488 (Life Technologies). Slides were mounted and imaged using a Zeiss Axiovert 200M outfitted with the ApoTome system (Carl Zeiss, Thornwood, NY).

\section{Statistical Analyses}

Results are presented as mean \pm SE. Prism 6.0 software (GraphPad Software, La Jolla, CA) was used to conduct analyses. A one-way ANOVA $(\alpha=0.05)$ followed by Tukey's post-hoc sorting was performed to determine significance between groups.

\section{Results}

Control tendons demonstrated the presence of M2 and occasional M1 macrophages in the endotenon layers, but no macrophages in the tendon fibers (Figure 2A). Three days following surgical tear and repair, M1 macrophages accumulated in regions of newly formed tendon tissue and remained present throughout the study period (Figure 2B-E). M2 macrophages slowly accumulated at sites of organizing tendon ECM and became the predominant macrophage phenotype by 28 days (Figure 2E). In most cases, after injury macrophages were localized in areas of tissue resorption as indicated by the lack of WGA staining. The change in M1 and M2 signal present in IHC was accompanied by quantitative changes in gene expression. For genes that regulate macrophage and neutrophil function (Figure 3), the neutrophil marker Ly6c was elevated by 3 days, but returned to levels similar to controls by 28 days. The pan-macrophage marker F4/80 was also elevated by 3 days. However, after an initial decline at 7 days, it remained elevated compared to controls thereafter. CCL2, which plays an important role in macrophage recruitment, was elevated by 3 days and then steadily declined over the next few weeks. Markers of M1 macrophages such as CD68, CCR7 and CD11b were dramatically elevated following surgical tear and repair. CD68 and CD11b declined between 3 and 7 days. CD168, a marker for M2 macrophages, remained similar to controls until day 28 at which time it became significantly elevated. Proinflammatory interleukins IL1b and IL6 were upregulated by 3 days, but IL6 demonstrated a gradual decline in expression between each time point. Upregulation of the antiinflammatory interleukin IL10 did not occur until 28 days.

For fibroblast proliferation and cell cycle control factors (Figure 4), the type II transmembrane protein tenomodulin (Tnmd) promotes tendon fibroblast proliferation and was significantly downregulated by 3 days. It was then elevated compared to controls by 7 days with peak expression at 14 days. Scleraxis (Scx), a bHLH transcription factor involved in tendon development, was elevated by 7 days with a slight decline in expression at 28 days. Alpha-smooth muscle actin (SMA) is an indicator of fibroblast contractility and was elevated at 7 days, but quickly returned to levels similar to controls by 14 days. No changes in the pan-fibroblast marker FSP1 were noted at any time point. Moreover, no differences in Mohawk (Mkx) expression, a marker of tendon maturation, were observed. HIF1a serves as 
a gauge of tissue hypoxia and was elevated by 3 days with further increases in expression at 7 and 14 days. Egr1 and Egr2, transcriptional regulatory proteins that are induced by various mitogenic cues, were elevated by 7 days. While Egr1 returned to levels similar to controls by 14 days, Egr 2 remained elevated for the remainder of the study period. The cellular differentiation marker Aatk remained similar to controls until day 28 at which time its expression was upregulated.

For EMT-related genes (Figure 5), the transcription factors Snail1 (Snai1) and Slug were elevated by 3 days and then declined by either 14 or 28 days, respectively. Goosecoid (Gsc), a homeobox protein that induces EMT, was elevated at 7 and 14 days compared to controls. Twist1, a bHLH transcription factor that also induces EMT, was elevated by 7 days and returned to baseline levels by 28 days. No differences in expression of the angiogenic factor VEGF or the transcription factor Foxc2, which is induced by EMT events, were noted at any time point. The endothelial cell marker CD31 was elevated at 7 days and the cell adhesion molecule E-cadherin (Cdh1) was significantly upregulated at 28 days. Ddr2, an EMTresponsive receptor tyrosine kinase, was downregulated by 3 days, but returned to levels similar to controls by 7 days.

For ECM gene expression (Figure 6), the small leucine-rich proteoglycan (SLRP) biglycan (Bgn) and the cartilage-specific proteoglycan aggrecan (Acan) were significantly elevated by 3 and 7 days, respectively. Both remained elevated with a slight decrease between 14 and 28 days. In contrast, the SLRPs decorin (Dcn) and fibromodulin (Fmod) were downregulated by 3 days, and only fibromodulin returned to levels similar to controls by 7 days. The intermediate filament vimentin (Vim) was briefly upregulated at 7 days, while the large proteoglycan versican (Vcan) was significantly expressed by 3 days and then steadily declined. Collagen type I (Col1a1) and type III (Col3a1) were both upregulated by 3 days with peak expression at 7 days. Members of the MMP family displayed similar levels of expression with upregulation of MMP2, MMP3, MMP8 and MMP14 by 3 days, and MMP9 by 7 days. MMP2, MMP8 and MMP14 were maximally expressed at 14 days and declined thereafter, while MMP9 steadily increased over the next few weeks. TIMP1 was elevated by 3 days and then declined by 14 days, whereas TIMP 2 was elevated by 7 days with peak expression at 14 days.

\section{Discussion}

While tendon injuries are common and can significantly detract from the quality of life, compared with other musculoskeletal tissues little is known about the biology of tendon repair. This was the first study, to our knowledge, that identified changes in canonical EMTrelated genes over the course of tendon injury and repair. Further, we report changes in different populations of macrophages, and fibroblast proliferation and ECM synthesis genes that play important structural and mechanical roles in tendon. There appears to be overlapping activation of various gene families within the first four weeks of tendon repair, and EMT-related factors may contribute to the repair process in injured tendon tissue.

In response to structural damage, chemotactic cytokines that promote the delivery of different cell populations to the repair site are released from tendon $\mathrm{ECM}^{3 ; 12}$. Phagocytic 
neutrophils and M1 macrophages are the first to arrive within 24 hours after injury, followed by a reparative shift in function that correlates with increases in M2 macrophages $1 ; 2 ; 5$. Using ED1 (CD68) and ED2 (CD163) to identify pro- and antiinflammatory macrophages, respectively, Marsolais and colleagues ${ }^{6}$ found that following the injection of collagenase into tendons, there was a rapid accumulation of M1 macrophages that returned to baseline levels two weeks after injury. No significant change in M2 macrophages was detected over the course of the study. In a study that explored early mechanical loading after collagenase injection into tendons, Godbout and colleagues ${ }^{21}$ reported an increase in M1 macrophages that was similar to Marsolais ${ }^{6}$, but also that exercise increased M2 macrophages three days after injury. The results of the current study are consistent with previous work as evidenced by upregulation of M1 markers CD68, CCR7 and CD11b as well as interleukins IL1b and IL6 within 72 hours. These proinflammatory factors remained consistently elevated at each time point, while the M2 marker CD168 and interleukin IL10 were not induced until 28 days. Similar observations in the early M1 response were found in injured skeletal muscle using a model of reloading after hindlimb suspension $22 ; 23$. Following resumption of weight bearing, M1 macrophages precipitously accumulate in the reloaded muscle within 48 hours, but expression was more transient with the M1 signal returning to levels similar to controls within one week post-injury. Although M2 macrophages also appeared at much earlier time points, the sequential transition between the M1 and M2 phenotypes supports the dual functionality of this cell type in the degradation and repair of injured tendons.

Following connective tissue injury, there is a pronounced early increase in the numbers of macrophages and fibroblasts ${ }^{1}$. While tendon fibroblasts are thought to come from either a circulating pool of bone marrow-derived progenitor cells or from resident populations of tendon stem/progenitor cells ${ }^{24}$, a growing body of evidence in other tissues suggests that resident cells in the host epithelium become activated and undergo an EMT transcriptional reprogramming to a mesenchymal fibroblast phenotype ${ }^{15 ; 16}$. As cells undergo EMT, they experience a loss of epithelial-associated junctional complexes, begin to express mesenchymal cell markers and acquire tissue-invasive properties that allow them to transmigrate their basement membrane and move into different environments. The epitenon is an epithelial structure subtended by a basement membrane ${ }^{14}$, and prior work in in vitro and in vivo models of lacerated flexor tendons has demonstrated that fibroblasts migrate from this outer layer into the site of tendon injury ${ }^{14 ; 25-27}$, as well as in response to treadmill training ${ }^{13}$. In the present study, we found that following acute Achilles tenotomy and repair there was a significant upregulation of canonical EMT-related genes including Snail1, Slug, Goosecoid and Twist1. These transcription factors repress cell adhesion proteins and induce metalloproteinases that degrade the ECM and promote tissue invasion ${ }^{28}$. Accompanying these changes were increased expression of mesenchymal tendon fibroblast markers scleraxis and tenomodulin, as well as ECM-remodeling enzymes MMP2, MMP3, MMP8, MMP9 and MMP14, and their endogenous inhibitors TIMP1 and TIMP2. Consistent with these results, other models of Achilles tendon injury have demonstrated massive increases in the expression of tendon-specific markers ${ }^{29 ; 30}$. Moreover, Scott and colleagues ${ }^{31}$ found that scleraxis, tenomodulin and type I collagen were significantly expressed in an injured mouse patellar tendon four weeks after a central defect was surgically created. Scleraxis and type I collagen expression eventually returned to 
baseline levels, but tenomodulin remained elevated even at 12 weeks post-injury. Induction of MMP expression has also been shown to correlate with collagen turnover and ECM remodeling during tendon repair $3 ; 32$. Further, proteoglycans are important regulators of collagen fibril assembly and variations in their content can greatly alter tendon structure and function ${ }^{33}$. Following surgical tear and repair of the Achilles tendon, we observed an upregulation of type I and III collagens, aggrecan, biglycan and versican, and a downregulation of decorin and fibromodulin. The reduction in decorin expression is supported by previous studies that demonstrated decreased rates of collagen fibrillogenesis in vitro in the presence of decorin ${ }^{34 ; 35}$. In addition to abnormal fibril structure and impaired mechanical function, decorin-deficient tendons display an increase in biglycan expression ${ }^{36}$. This may compensate for the loss of decorin as biglycan is expressed during early tendon development, but its expression normally declines as the tendon matures ${ }^{37}$. Similar to the altered tendon phenotype described in decorin-null mice, a deficiency of fibromodulin also produces tendons with thin, irregular collagen fibrils ${ }^{38}$. Aggrecan is a proteoglycan that is expressed during later stages of chondrogenesis, and is also expressed in regenerating tendons following tenotomy ${ }^{39}$. Increased expression of aggrecan is correlated with the induction of heterotopic ossification in injured tendons ${ }^{39}$, and although we did not directly measure other markers of osteogenesis, the increase in aggrecan at later time points suggests some heterotopic ossification was also occurring in our model. Overall, findings from this study in conjunction with previous work suggest that the assembly of the collagen network during tendon repair may be mediated by the temporal-specific expression of proteoglycans.

This study provided important insights into changes in macrophage phenotype and EMTrelated genes following acute Achilles tenotomy and repair, but there are several limitations. We did not immobilize the hindlimbs postoperatively, which is standard practice in patients undergoing Achilles tendon repairs. However, the plantaris tendon was left intact, and as plantaris is substantially larger in rats than humans, this likely provides stress shielding for the injured Achilles tendon. Immediate mobilization at the repair site may have impacted the amount of inflammation present, but should not have altered the sequence of inflammatory cell accumulation within the injured tissue. We also focused only on the Achilles tendon and observed differences may not be reflected in trunk or other limb tendons. While there are 26 known MMP genes ${ }^{3}$, we evaluated only a subset of MMPs that were selected from the four general classes of MMPs. Our animal model is most representative of acute tendon transections, and may not recapitulate longstanding degenerative changes in the tendon ECM. Finally, in addition to driving transdifferentiation and migration through the EMT program, Snail1, Slug, Goosecoid and Twist1 have other EMT-independent functions including cell lineage specification, cell cycle regulation, apoptosis, and both ECM synthesis and remodeling $40-43$. While these results are encouraging, whether EMT is truly occurring in tendon, or fibroblasts emerging from the epitenon are simply proliferating populations of resident cells, remains to be determined. Further studies are needed to decipher the specific roles of these genes during tendon repair. This study evaluated changes in the expression of EMT-related genes during the repair of injured tendon tissue, but changes in the levels of specific proteins were not investigated. However, previous studies in other tissues have 
demonstrated that changes in the expression of specific EMT-related genes correlate with changes in the abundance of the translated proteins ${ }^{44 ; 45}$.

Injuries to and diseases of tendons are a substantial economic and health burden among patients of all ages. Nonoperative interventions focus on symptomatic relief, and even with surgery patients may have poor outcomes and continue to experience pain and reduced function ${ }^{1}$. A more direct approach to the management of tendon injuries is needed, and the current lack of targeted therapies is due in part to our limited mechanistic insight into basic physiological processes that control tendon repair. Findings from this study and from Marsolais and colleagues ${ }^{6}$ demonstrate that macrophages change phenotype in response to tendon damage and this likely contributes to the degradation and subsequent repair of injured tendon ECM. Further investigation of the specific functions of macrophage subpopulations in tendon is therefore warranted. Lastly, given the critical role EMT plays in the postnatal growth of many tissues in the body and in pathologic conditions such as tissue fibrosis, the expression of EMT-related genes during tendon repair may prove to be a worthwhile area of scientific exploration.

\section{Supplementary Material}

Refer to Web version on PubMed Central for supplementary material.

\section{Acknowledgments}

This work was supported by NIH grants R01-AR063649, T32-GM008616 and T32-GM008322.

\section{References}

1. Sharma PP, Maffulli NN. Tendon injury and tendinopathy: healing and repair. J Bone Joint Surg Am. 2005; 87:187-202. [PubMed: 15634833]

2. Voleti PB, Buckley MR, Soslowsky LJ. Tendon Healing: Repair and Regeneration. Annu Rev Biomed Eng. 2012; 14:47-71. [PubMed: 22809137]

3. Davis ME, Gumucio JP, Sugg KB, et al. MMP inhibition as a potential method to augment the healing of skeletal muscle and tendon extracellular matrix. Journal of Applied Physiology. 2013; 115:884-891. [PubMed: 23640595]

4. Jarvinen M, Jozsa L, Kannus P, et al. Histopathological findings in chronic tendon disorders. Scandinavian journal of medicine \& science in sports. 1997; 7:86-95. [PubMed: 9211609]

5. Hope M, Saxby TS. Tendon healing. Foot and ankle clinics. 2007; 12:553-567. v. [PubMed: 17996614]

6. Marsolais D, Côté CH, Frenette J. Neutrophils and macrophages accumulate sequentially following Achilles tendon injury. J Orthop Res. 2001; 19:1203-1209. [PubMed: 11781025]

7. Fukasawa M, Bryant SM, Nakamura RM, et al. Modulation of fibroblast proliferation by postsurgical macrophages. The Journal of surgical research. 1987; 43:513-520. [PubMed: 3695452]

8. Leadbetter WB. Cell-matrix response in tendon injury. Clin Sports Med. 1992; 11:533-578. [PubMed: 1638640]

9. Laskin DL. Macrophages and inflammatory mediators in chemical toxicity: a battle of forces. Chemical research in toxicology. 2009; 22:1376-1385. [PubMed: 19645497]

10. Sica A, Mantovani A. Macrophage plasticity and polarization: in vivo veritas. The Journal of clinical investigation. 2012; 122:787-795. [PubMed: 22378047]

11. Mann CJ, Perdiguero E, Kharraz Y, et al. Aberrant repair and fibrosis development in skeletal muscle. Skeletal muscle. 2011; 1:21. [PubMed: 21798099] 
12. Kjaer M. Role of Extracellular Matrix in Adaptation of Tendon and Skeletal Muscle to Mechanical Loading. Physiological Reviews. 2004; 84:649-698. [PubMed: 15044685]

13. Mendias CL, Gumucio JP, Bakhurin KI, et al. Physiological loading of tendons induces scleraxis expression in epitenon fibroblasts. J Orthop Res. 2011; 30:606-612. [PubMed: 21913219]

14. Taylor SH, Al-Youha S, Van Agtmael T, et al. Tendon Is Covered by a Basement Membrane Epithelium That Is Required for Cell Retention and the Prevention of Adhesion Formation. PLoS ONE. 2011; 6:e16337. [PubMed: 21298098]

15. Iwano M, Plieth D, Danoff TM, et al. Evidence that fibroblasts derive from epithelium during tissue fibrosis. J Clin Invest. 2002; 110:341-350. [PubMed: 12163453]

16. Kalluri R, Weinberg RA. The basics of epithelial-mesenchymal transition. J Clin Invest. 2009; 119:1420-1428. [PubMed: 19487818]

17. Lee JM. The epithelial-mesenchymal transition: new insights in signaling, development, and disease. The Journal of Cell Biology. 2006; 172:973-981. [PubMed: 16567498]

18. Schmittgen TD, Livak KJ. Analyzing real-time PCR data by the comparative C(T) method. Nature protocols. 2008; 3:1101-1108.

19. Chin LK, Calabro A, Rodriguez ER, et al. Characterization of and host response to tyramine substituted-hyaluronan enriched fascia extracellular matrix. Journal of Materials Science: Materials in Medicine. 2011:1-13. [PubMed: 21052792]

20. Badylak SF, Valentin JE, Ravindra AK, et al. Macrophage phenotype as a determinant of biologic scaffold remodeling. Tissue engineering Part A. 2008; 14:1835-1842. [PubMed: 18950271]

21. Godbout C, Ang O, Frenette J. Early voluntary exercise does not promote healing in a rat model of Achilles tendon injury. J Appl Physiol (1985). 2006; 101:1720-1726. [PubMed: 16916920]

22. St Pierre BA, Tidball JG. Macrophage activation and muscle remodeling at myotendinous junctions after modifications in muscle loading. Am J Pathol. 1994; 145:1463-1471. [PubMed: 7992849]

23. Tidball JG. Mechanisms of muscle injury, repair, and regeneration. Comprehensive Physiology. 2011; 1:2029-2062. [PubMed: 23733696]

24. Bi Y, Ehirchiou D, Kilts TM, et al. Identification of tendon stem/progenitor cells and the role of the extracellular matrix in their niche. Nat Med. 2007; 13:1219-1227. [PubMed: 17828274]

25. Gelberman RH, Steinberg D, Amiel D, et al. Fibroblast chemotaxis after tendon repair. J Hand Surg Am. 1991; 16:686-693. [PubMed: 1880367]

26. Jones ME, Mudera V, Brown RA, et al. The early surface cell response to flexor tendon injury. J Hand Surg Am. 2003; 28:221-230. [PubMed: 12671852]

27. Manske PR, Lesker PA. Histologic evidence of intrinsic flexor tendon repair in various experimental animals. An in vitro study. Clin Orthop Relat Res. 1984:297-304. [PubMed: 6420101]

28. Peinado H, Olmeda D, Cano A. Snail, Zeb and bHLH factors in tumour progression: an alliance against the epithelial phenotype? Nat Rev Cancer. 2007; 7:415-428. [PubMed: 17508028]

29. Eliasson P, Andersson T, Aspenberg P. Rat Achilles tendon healing: mechanical loading and gene expression. J Appl Physiol (1985). 2009; 107:399-407. [PubMed: 19541731]

30. James R, Kesturu G, Balian G, et al. Tendon: Biology, Biomechanics, Repair, Growth Factors, and Evolving Treatment Options. J Hand Surg Am. 2008; 33:102-112. [PubMed: 18261674]

31. Scott A, Sampaio A, Abraham T, et al. Scleraxis expression is coordinately regulated in a murine model of patellar tendon injury. J Orthop Res. 2010; 29:289-296. [PubMed: 20740671]

32. Riley G. The pathogenesis of tendinopathy. A molecular perspective. Rheumatology. 2004; 43:131-142. [PubMed: 12867575]

33. Dunkman AA, Buckley MR, Mienaltowski MJ, et al. Decorin expression is important for agerelated changes in tendon structure and mechanical properties. Matrix biology: journal of the International Society for Matrix Biology. 2013; 32:3-13. [PubMed: 23178232]

34. Vogel KG, Paulsson M, Heinegard D. Specific inhibition of type I and type II collagen fibrillogenesis by the small proteoglycan of tendon. The Biochemical journal. 1984; 223:587-597. [PubMed: 6439184] 
35. Danielson KG, Baribault H, Holmes DF, et al. Targeted disruption of decorin leads to abnormal collagen fibril morphology and skin fragility. J Cell Biol. 1997; 136:729-743. [PubMed: 9024701]

36. Zhang G, Ezura Y, Chervoneva I, et al. Decorin regulates assembly of collagen fibrils and acquisition of biomechanical properties during tendon development. Journal of cellular biochemistry. 2006; 98:1436-1449. [PubMed: 16518859]

37. Ansorge HL, Adams S, Birk DE, et al. Mechanical, compositional, and structural properties of the post-natal mouse Achilles tendon. Annals of biomedical engineering. 2011; 39:1904-1913. [PubMed: 21431455]

38. Svensson L, Aszodi A, Reinholt FP, et al. Fibromodulin-null mice have abnormal collagen fibrils, tissue organization, and altered lumican deposition in tendon. J Biol Chem. 1999; 274:9636-9647. [PubMed: 10092650]

39. Lin L, Shen Q, Xue T, et al. Heterotopic ossification induced by Achilles tenotomy via endochondral bone formation: expression of bone and cartilage related genes. Bone. 2010; 46:425-431. [PubMed: 19735753]

40. Barrallo-Gimeno A. The Snail genes as inducers of cell movement and survival: implications in development and cancer. Development. 2005; 132:3151-3161. [PubMed: 15983400]

41. Rowe RG, Li XY, Hu Y, et al. Mesenchymal cells reactivate Snaill expression to drive threedimensional invasion programs. The Journal of Cell Biology. 2009; 184:399-408. [PubMed: 19188491]

42. Sekiya SS, Suzuki AA. Glycogen synthase kinase $3 \beta$-dependent Snail degradation directs hepatocyte proliferation in normal liver regeneration. Proc Natl Acad Sci USA. 2011; 108:1117511180. [PubMed: 21690373]

43. Vega S. Snail blocks the cell cycle and confers resistance to cell death. Genes \& Development. 2004; 18:1131-1143. [PubMed: 15155580]

44. Rowe RG, Lin Y, Shimizu-Hirota R, et al. Hepatocyte-Derived Snail1 Propagates Liver Fibrosis Progression. Mol Cell Biol. 2011; 31:2392-2403. [PubMed: 21482667]

45. Medici D, Hay ED, Olsen BR. Snail and Slug promote epithelial-mesenchymal transition through beta-catenin-T-cell factor-4-dependent expression of transforming growth factor-beta3. Mol Biol Cell. 2008; 19:4875-4887. [PubMed: 18799618] 


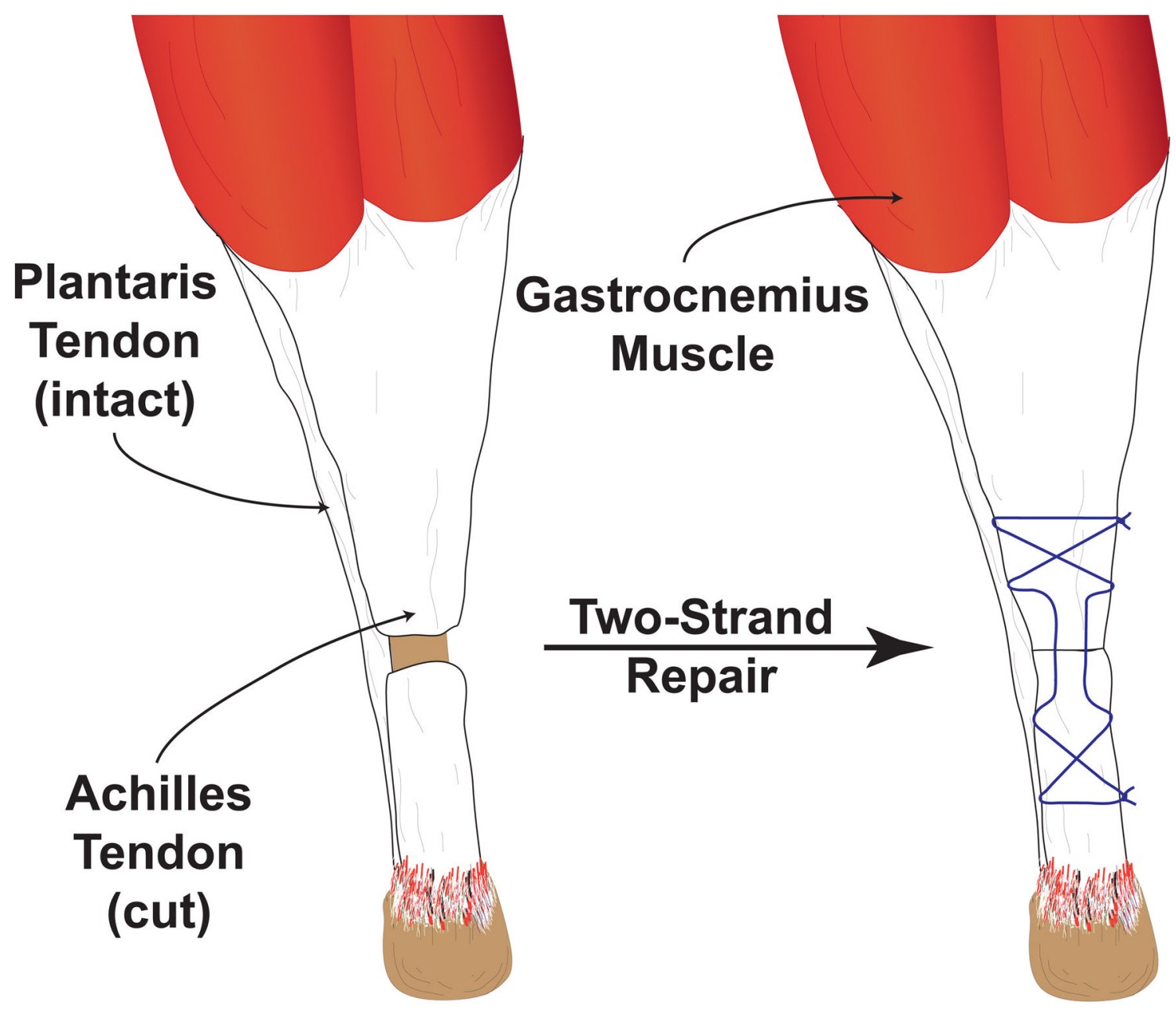

Figure 1.

Illustration of tendon repair. A full-thickness tenotomy is performed in the midsubstance of the Achilles tendon and the plantaris tendon is left intact. The defect is then repaired immediately using a two-strand core suture (Bunnell technique). 

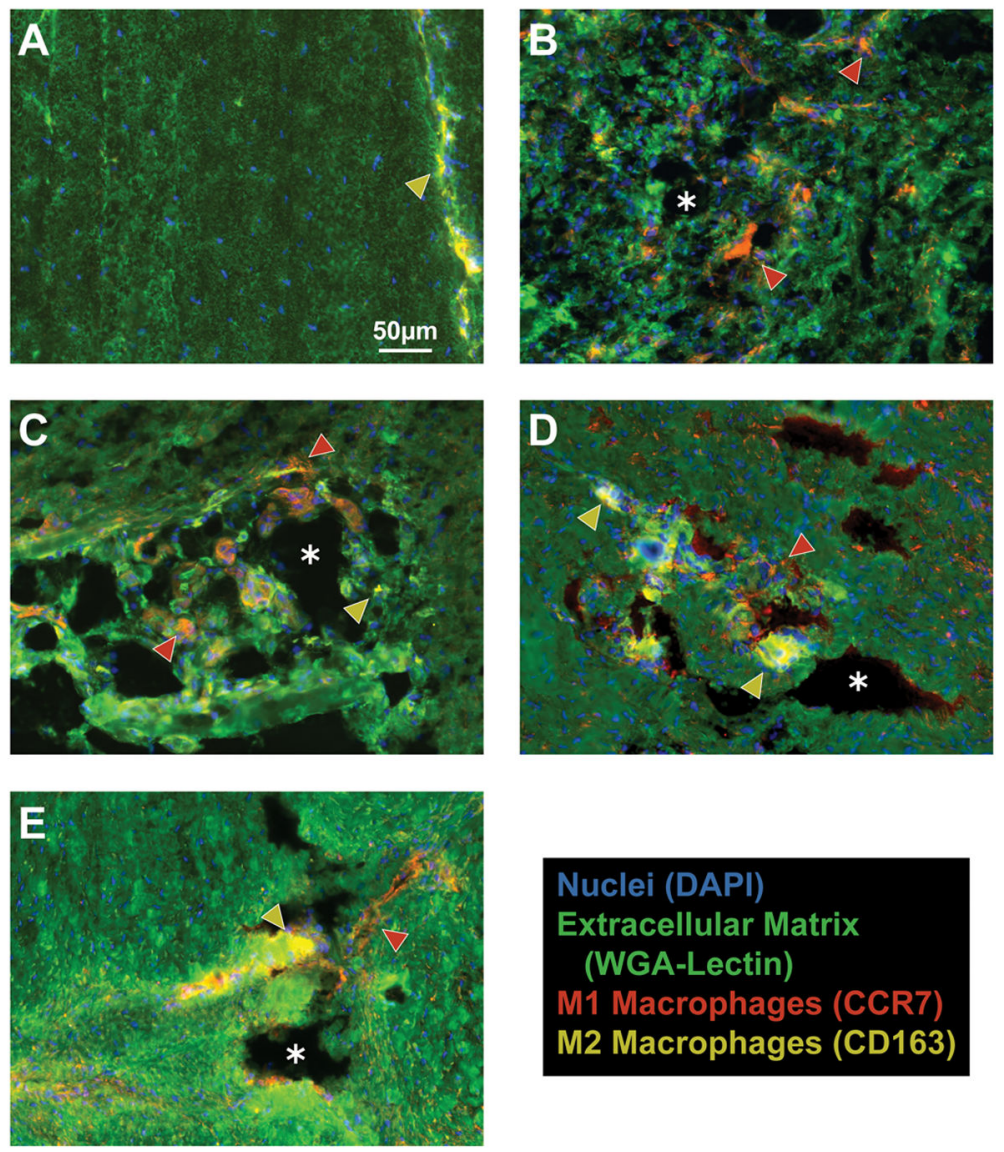

Figure 2.

Macrophages accumulate sequentially in tendon following surgical tear and repair. M2 macrophages are found in the endotenon of non-operated controls $(\mathrm{A})$, but not in the tendon fibers. There is a dramatic accumulation of M1 macrophages in regions of ECM resorption that remained consistent in the 3-day (B), 7-day (C), and 14-day (D) groups. M2 macrophages become the predominant phenotype by 28 days (E). M1 macrophages (CCR7), red (and indicated by red arrowheads); M2 macrophages (CD163), yellow (and indicated by yellow arrowheads); ECM (WGA-lectin), green; nuclei (DAPI), blue. Asterisks indicate areas of tissue resorption. All panels are shown at the same level of magnification as in panel A. 


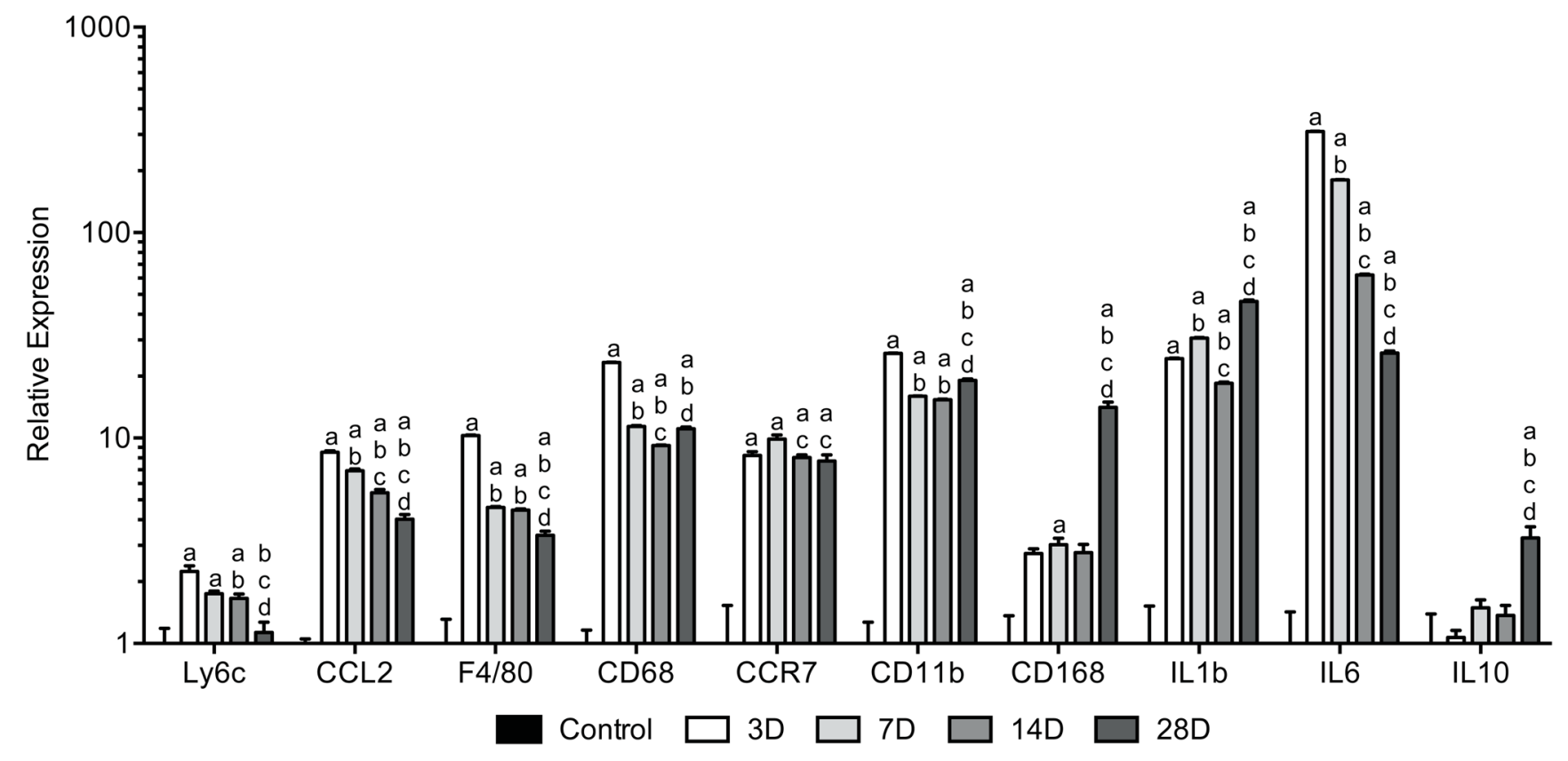

Figure 3.

Changes in gene expression of macrophage and neutrophil markers in Achilles tendon following surgical tear and repair. Target genes were normalized to B2M expression, and further normalized to the control group. Differences were tested using a one-way ANOVA ( $a=0.05$ ) followed by Tukey's post-hoc sorting. a, different from Control; $b$, different from 3D; c, different from 7D; d, different from 14D. N=6 tendons per group. 


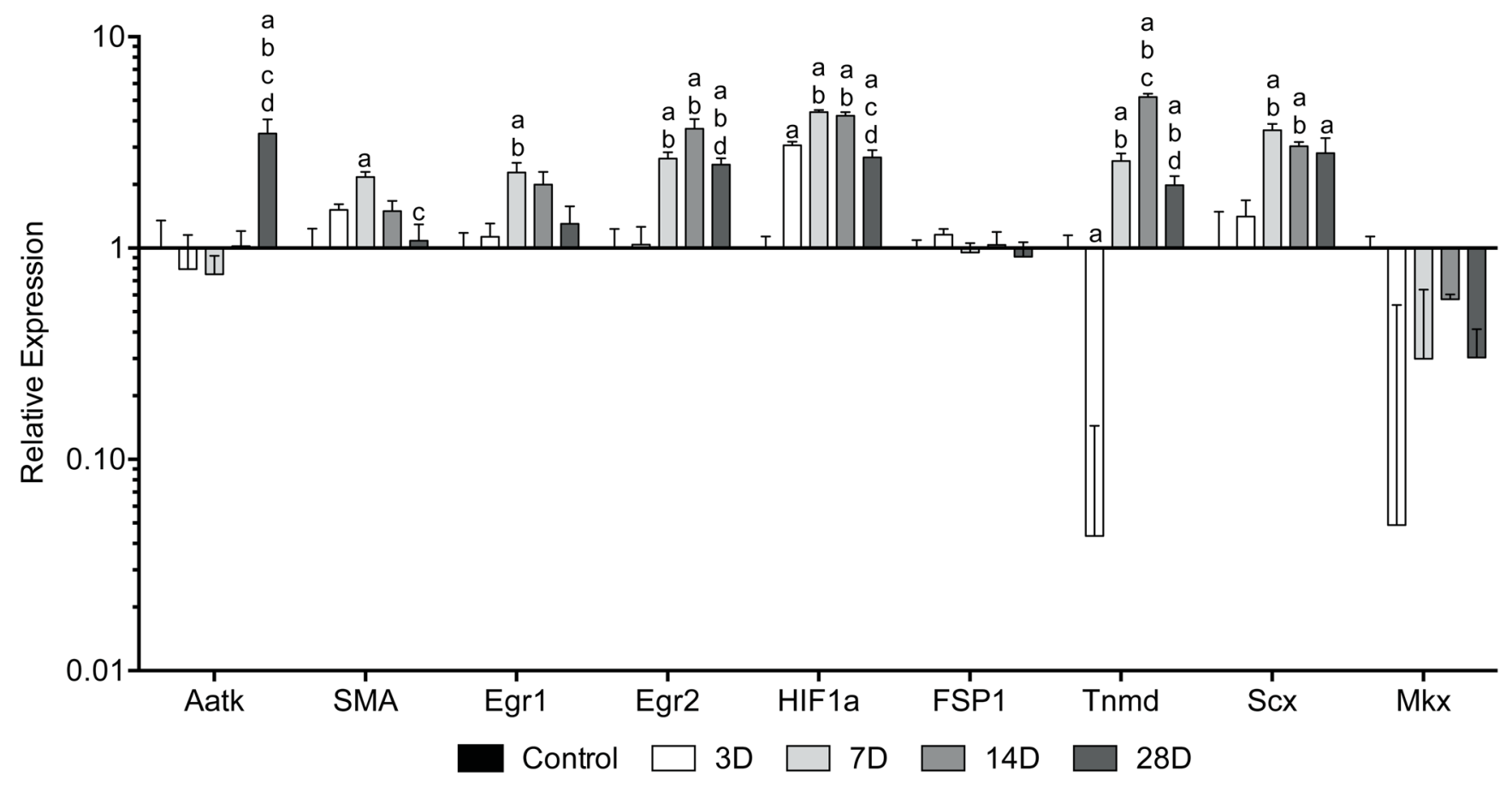

Figure 4.

Changes in gene expression of fibroblast proliferation and cell cycle control factors in Achilles tendon following surgical tear and repair. Target genes were normalized to B2M expression, and further normalized to the control group. Differences were tested using a oneway ANOVA ( $a=0.05$ ) followed by Tukey's post-hoc sorting. a, different from Control; b, different from 3D; c, different from 7D; d, different from 14D. N=6 tendons per group. 


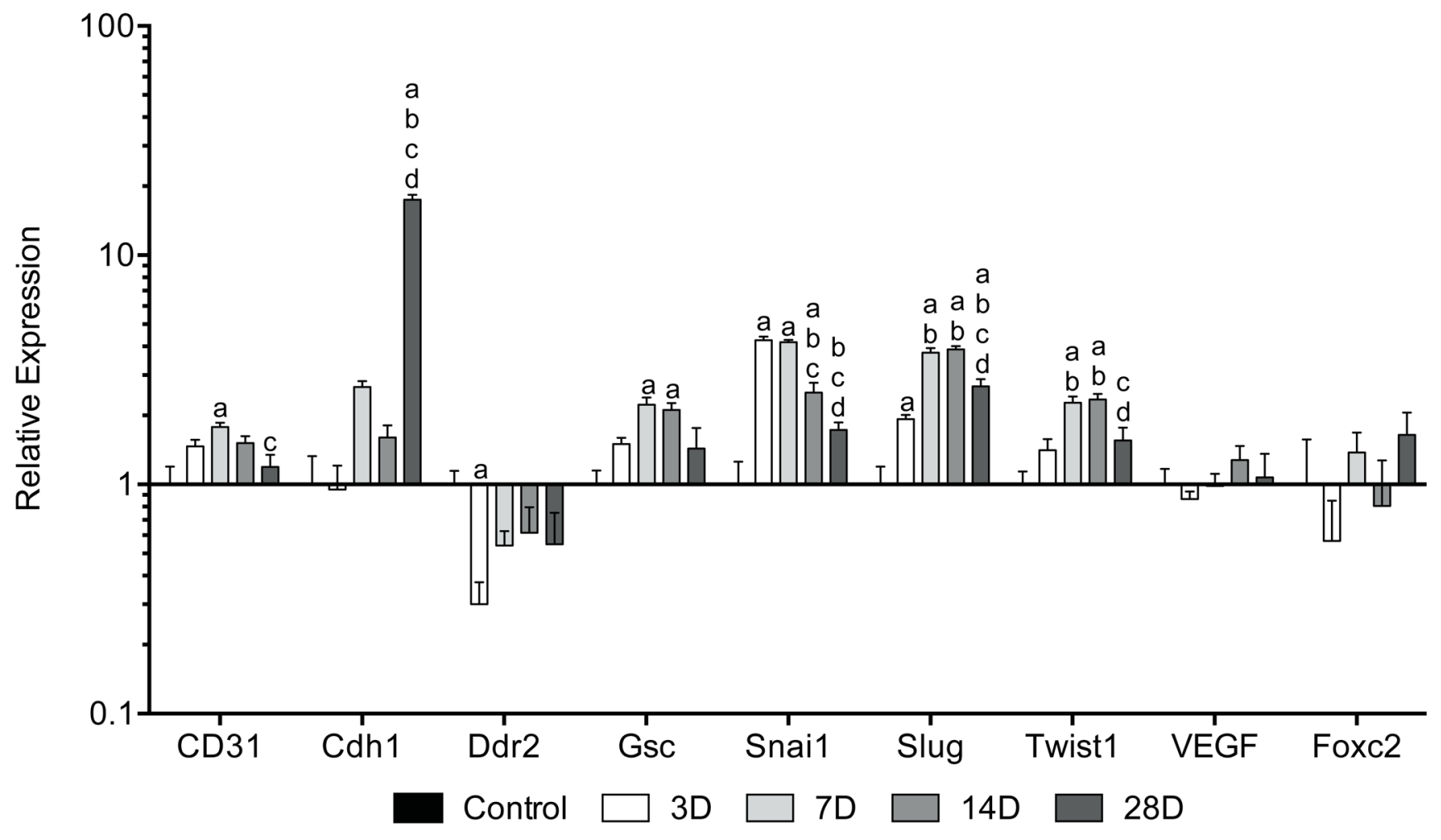

Figure 5.

Changes in gene expression of EMT-related genes in Achilles tendon following surgical tear and repair. Target genes were normalized to $\mathrm{B} 2 \mathrm{M}$ expression, and further normalized to the control group. Differences were tested using a one-way ANOVA $(a=0.05)$ followed by Tukey's post-hoc sorting. a, different from Control; b, different from 3D; c, different from 7D; d, different from 14D. N=6 tendons per group. 


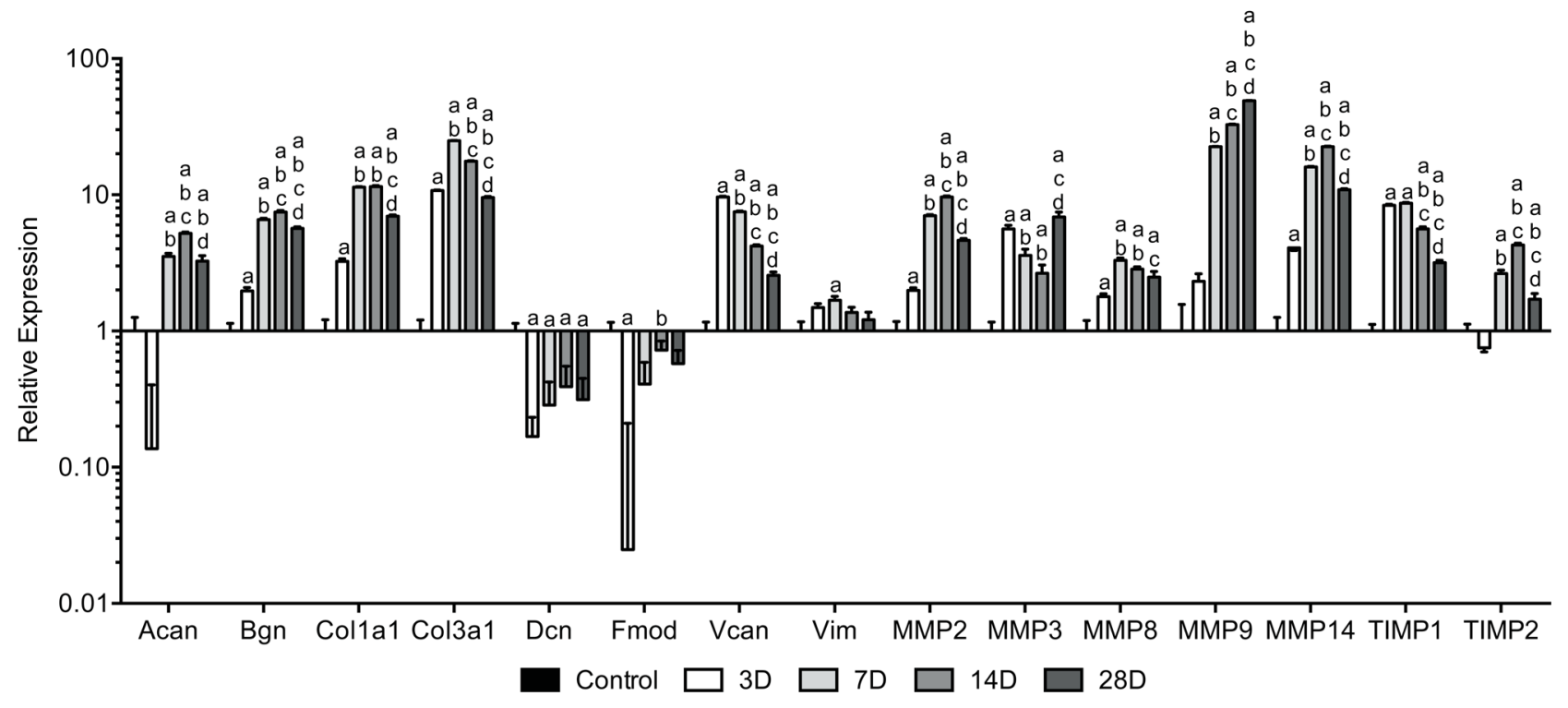

Figure 6.

Changes in gene expression of ECM components and MMPs in Achilles tendon following surgical tear and repair. Target genes were normalized to B2M expression, and further normalized to the control group. Differences were tested using a one-way ANOVA $(a=0.05)$ followed by Tukey's post-hoc sorting. a, different from Control; b, different from 3D; c, different from 7D; d, different from 14D. $\mathrm{N}=6$ tendons per group. 